\title{
Audience Participation Fighting Game: making an APG using a concept of social facilitation
}

This paper was downloaded from TechRxiv (https://www.techrxiv.org).

\section{LICENSE}

CC BY 4.0

SUBMISSION DATE / POSTED DATE

18-02-2022 / 23-02-2022

\section{CITATION}

Paliyawan, Pujana; Thawonmas, Ruck; Sookhanaphibarn, Kingkarn; Choensawat, Worawat (2022): Audience Participation Fighting Game: making an APG using a concept of social facilitation. TechRxiv. Preprint. https://doi.org/10.36227/techrxiv.19193666.v1

$\mathrm{DOI}$

10.36227/techrxiv.19193666.v1 


\title{
Audience Participation Fighting Game: making an APG using a concept of social facilitation
}

\author{
Pujana Paliyawan*, Ruck Thawonmas ${ }^{\dagger}$, Kingkarn Sookhanaphibarn ${ }^{\ddagger}$, Worawat Choensawat \\ *Research Organization of Science and Technology, Ritsumeikan University, Japan \\ ${ }^{\dagger}$ College of Information Science and Engineering, Ritsumeikan University, Japan \\ ${ }^{\ddagger}$ Multimedia Intelligent Technology Lab, Bangkok University, Thailand \\ ruck@is.ritsumei.ac.jp
}

\begin{abstract}
This paper presents architecture and design of a Twitch-based audience participation fighting game, where the strengths of game AI characters are dynamically adjusted based on audience cheering and jeering. Limitations underlying existing audience participation games (APGs) are discussed, and followed by solutions as demonstrated in our game. Our study includes AI tests on 500000 one-minute game rounds and a user experiment with 82 participants. We investigated user experience (UX), comparing two different kinds of gameplays (collaborative vs competitive), as well as two different kinds of interaction (cheering vs jeering). A pairwise preference, four-alternative forced choice (4-AFC), version of the Game User Experience Satisfaction Scale (GUESS) is used for UX assessment. At the end, we provided guidelines and hypotheses for future APG research.

Index Terms-audience participation games, game live streaming, game AI, HCI
\end{abstract}

\section{INTRODUCTION}

Watching live streaming video games has been immensely popular [13]. It is mentioned in 2017 [5], that Twitch has gathered a substantial world wide community and brought an evolution called "user-generated live streaming," or to say unlike other platforms, it often involves things like live social interaction. From then until this 2021, the number of Twitch broadcasters and users has increased more rapidly to 9 and 2.84 millions-about 4 times from 20171 . A dramatic growth was found during COVID-19 pandemic, in the era of social distancing.

Recently, World Health Organization (WHO) reported that COVID-19 has seeded a major mental health crisis ${ }^{2}$; people are distressed due to immediate health impacts and the consequences of isolation. To look after mental health, WHO suggests social contact via online channels, games, and social media. Considering that Twitch enables community building by affording social connectivity, social interactivity, and further content discovery [24], and that live-streams can act as virtual places, establishing communities with a multidimensional ecosystem of relationships between streamer and audiences [29], we believe live streaming games are now, not only a type of entertainment, but also a potential solution to help people cope with psychological problems in this era.

\footnotetext{
${ }^{1}$ https://www.businessofapps.com/data/twitch-statistics/

${ }^{2}$ www.who.int/campaigns/connecting-the-world-to-combatcoronavirus/healthyathome/healthyathome-mental-health
}

Beyond live streaming games, Audience Participation Games (APGs) take a further step in promoting social connectivity by incorporating massive audiences into gameplay [23]. They blur the line between audiences and players by allowing the audiences to impact gameplay [23], [25]. Engaging viewers has the power to create authentic social connections, turning one-direction interactions into bidirectional social experiences, akin to "seeing friends" [30]. Audience participation is affected not only by the immersiveness of content, but by the immediacy of interactions and the sociality of the experience [9]. These interactions are not simply social, but can become integral elements of a play experience [4]. In this socialdistancing era, where people are turning to live-streaming as a substitute for rich in-person experiences, APGs offer a new way for audiences to engage, yet the potential design spaces have not been adequately described [25].

This paper presents the design and architecture of an audience participation fighting game. The game system is designed based on a concept of social facilitation [35], the phenomenon when an individual performs better, or worse, on tasks when others are around; more precisely, the game has AI characters whose strengths are dynamically adjusted based on audience inputs. Methods to overcome limitations in existing APGs are presented. We further investigate areas that have not yet been well-studied, namely factors affecting user experience (UX) and activeness in an APG environment. Some discoveries reveal how different kinds of gameplays (competitive and collaborative) and different kinds of interaction (cheering and jeering) likely affect APGs. Some contributions in this paper are given below.

- The first audience participation fighting game, implemented without modifying the game, but the game's AI.

- Design and architecture for low-cost APGs that overcome limitations such as a limited quota for data sent, a messy chat channel, and the need of a Twitch account.

- The first study, on differences in UX and activeness, comparing between competitive and competitive game, as well as cheering and jeering, under APG environment; results provide directions and hypotheses for future research.

- A 4-AFC version of GUESS [21], a questionnaires for UX assessment based on pairwise preference protocol [32], [33], [3]. 


\section{Literature Review}

Previous studies on social facilitation are first summarized. Existing APGs are then introduced with discussions on their limitations. Previous works on audience experience and game designs are mentioned. Finally, details about FightingICE and previous studies on it are given. Table I shows a summary of comparison.

\section{A. Social facilitation}

Social facilitation is a long known concept. In the late 18th century, Norman Triplett [26] found that cyclists ride faster when they were racing with other riders, so he conducted a study [27] and found that individual performance can be affected by the presence of others-this became a landmark paper on social facilitation. Many researchers followed his findings and investigated how the presence of others impacts individual performance, not only as competitors, but also as observers and audiences. In 1920, the terms "social facilitation" was defined by Floyd Allport [2] as a tendency for individuals to perform differently when in the mere presence of others.

For social facilitation in the context of games, in 2014, Kappen et al. [12] claimed their study as the first study of the relevance of co-located audience influence on player experience. There have been studies following this study, but to the best of our knowledge, there is not yet a study on APGs. Most of these studies also focus on the player aspect. For example, Kimble et al [14] studied player performance under audience scrutiny; they reported that on the complex game, players performed worse when they were being observed. Bowman et al. [1] reported an increase of performance of players playing an easy version of an FPS game in the presence of a real audience. Lin et al. [16] studied social interactions between audiences and players in an environment of a game arcade center. Emmerich et al. [8] investigated the impact of real observers and virtual agents on player experience and performance in four different games. It is also noted that many studies used a controlled set of audiences (e.g., [12], [14], [16]), some of which were experimenters, not real audiences.

Social facilitation is a core idea inspiring our proposed APG. In our fighting game, there are two players fighting against each other; each of which can be either human or AI. We present an AI whose strength is dynamically adjusted based on audience inputs. Our system is tested on a big group of audiences under a real streaming environment. We also plan to conduct a study on social facilitation using one human player fighting against an $\mathrm{AI}$ in the future.

\section{B. Audience Participation Games (APGs)}

One early study on APGs was by Seering et al. [23], where two prototype games (FPS and Racing) were presented with four variants (methods of interactions) each. Consequentially, Twitch Plays Pokémon [22] became a notable example, and the success of this game inspires other APGs, such as Legend of Dungeon: Masters ${ }^{3}$, an APG in which audiences can help

${ }^{3}$ https://store.steampowered.com/app/405980/Legend_of_Dungeon_Masters/ the player by sending mighty weapons or, alternatively, raining upon the player beasts and demons, and JUSTIN [18], a serious APG developed for collecting image description data via game live streaming.

One limitation underlying most APGs is that they send data via chat channel, which has a limit on the number of messages allowed and also results in a messy chat room; we solve this problem by proposing a new architecture employing a web server with an SQL database. In addition, existing works on APG did not discuss on effects from different kinds of interaction.

\section{Audience Experience}

Some recent studies focused on the experience of the audience as a significant part of the social gaming situation as the following examples. Downs et al. [6], [7] argued that audiences are frequently a part of social gaming situations, but that their experiences are not well understood, and mentioned that the experience of audience members changes when they are informed that the gaming session involves turn-taking rather than mere observation. Lessel et al. [15] expanded video game live-streams with enhanced communication channels; it was claimed to improve the communication, give the audience a feeling of influence, and raise their activity level; however, their design is for specific turn-based games. Maurer et al. [17] conducted a study using a game with gaze detection, comparing three condition: (1) no observer input, (2) visualizing the gaze-point of an observer within the game, (3) observer's gaze acts as a flashlight helping the player uncovers the greyed out areas. They report that gaze-based onlooker integration changes the gaming experience of both player and audience. Our work is similar to these ones in the way that we focus on UX, but we compare different designs for participation.

\section{Designs for participation}

Audience participation can be done in various forms. Those considered in our study are as follows:

Cheer vs Jeer: Kappen et al. [12] studied differences in game experience of players under an environment with silent, positive, and negative audiences. However, their study is in a non-APG environment (no audience input to the game), audiences were co-located, comments for positive and negative audiences were scripted, and the study focuses on the player. On the other hand, our work focuses on UX of APG audiences in a real streaming environment.

Competitive vs Collaborative: Wehbe et al. [28] investigated whether social conditions (i.e., social cooperative, competitive, multiplayer environments, or computer-controlled single-player environments) are comparable, or if there are conditions that affect the player experience more significantly. However, the study was conducted in a co-located environment. There were only 2 players, and no audience, in their tested game. 
TABLE I

COMPARISON OF THE PROPOSED WORK TO EXISTING WORKS OVER SEVEN dimensions: [A] Social Facilitation, [B] Many Audiences (>2), [C] APG (TAKe Audience InPUTS), [D] STUdy AudienCe Experience, [E] Cheer vs Jeer, [F] Competitive vs Collaborative, [G] AI

\begin{tabular}{|c|c|c|c|c|c|c|c|}
\hline \multirow{2}{*}{ Comparison works } & \multicolumn{3}{|c|}{ APG-related } & \multicolumn{3}{|c|}{ Game-UX-related } & AI \\
\cline { 2 - 7 } [1], [2], [14], [26], [27] & $\checkmark$ & X & X & X & X & X & X \\
[12] & $\checkmark$ & $\checkmark$ & X & $\checkmark$ & $\checkmark$ & X & X \\
[16] & $\checkmark$ & $\checkmark$ & X & X & X & X & X \\
[8] & $\checkmark$ & X & X & X & X & X & $\checkmark$ \\
[22], [23] & X & $\checkmark$ & $\checkmark$ & X & X & X & X \\
[18] & X & $\checkmark$ & $\checkmark$ & $\checkmark$ & X & X & X \\
[6], [7] & - & X & X & $\checkmark$ & X & X & X \\
[15] & - & $\checkmark$ & $\checkmark$ & $\checkmark$ & X & X & X \\
[17] & $\checkmark$ & X & $\checkmark$ & $\checkmark$ & X & X & X \\
[28] & - & X & X & X & X & $\checkmark$ & $\checkmark$ \\
[34] & X & X & X & X & X & X & $\checkmark$ \\
[19] & $\checkmark$ & X & $\checkmark$ & X & X & X & $\checkmark$ \\
Proposed system & $\checkmark$ & $\checkmark$ & $\checkmark$ & $\checkmark$ & $\checkmark$ & $\checkmark$ & $\checkmark$ \\
\hline \multicolumn{7}{|c}{$\checkmark:$ yes, X: no, -: unclear or undefined }
\end{tabular}

\section{E. FightingICE and AIs}

FightingICE ${ }^{4}$ is an open-source fighting game AI development platform that has been used for running many AI competitions. Several winning AIs were MCTS-based, and there have been many MCTS-based AIs presented. One example is MctsAi by Yoshida et al. [34] publicly available on the FightingICE website ${ }^{5}$.

Recently, our group attempted to make an APG version of FightingICE by implementing a generalized Believable Entertaining AI (gBEAI) [19] that generates entertaining gameplay by dynamic difficulty adjustment while maintaining believable behavior. The strength of this MCTS-based AI is dynamically adjusted according to a given social facilitation parameter $F$ that has value is in a range of $[-1,1] ;-1$ makes the $\mathrm{AI}$ aim to win against the opponent by a hit-point (HP) difference of $120^{6}$, and 1 makes the AI target to lose the opponent by an HP difference of 120 .

The previous work above only tested the performance of the $\mathrm{AI}$ in terms of strength adjustment and evaluated its behavior in terms of believability; $F$ was programatically set for testing. In this study, $F$ is dynamically adjusted based on cheering and jeering actions from audiences. Namely, audiences can click a cheer button to strengthen an AI of their team, and click a jeer button to weaken the opponent AI.

${ }^{4}$ https://www.ice.ci.ritsumei.ac.jp/ ftgaic/index-R.html

${ }^{5}$ http://www.ice.ci.ritsumei.ac.jp/\%7eftgaic/index-2h.html

6the value of 120 is set based on the damage incurred by a final blow skill

6https://handy-games.com/en/games/one-hand-clapping/

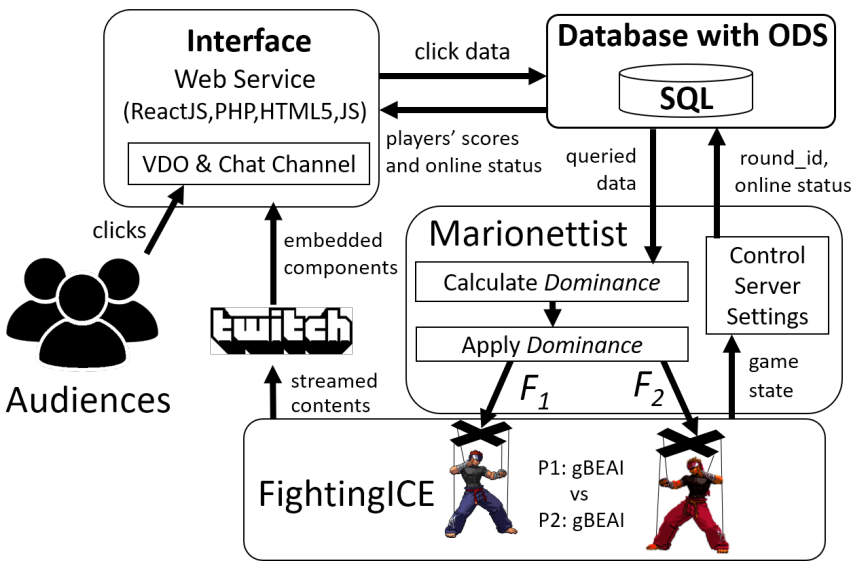

Fig. 1. The architecture of the game system.

\section{Proposed System}

In the proposed APG, each user joins either Team P1 or $\mathrm{P} 2$, and supports the AI of their team by pressing buttons; they get scores from participating, and the names of top-10 users with the highest scores are shown on a leaderboard. In Competitive Game, users are divided into two sides (Team P1 and Team P2), while in Collaborative Game, all the users are on the same side (Team P1). The architecture of the game system is shown in Fig. 1; there are four major components, whose details are given in subsections.

\section{A. Interface}

The interface is a web application running on our web server. When a user visits the index page, he/she will be asked to $\log$ in to enter the system. In an official version, it is required to have an account, which can be registered on our site (implemented based on Firebase authentication; email verification is required; a Twitch account is not needed). However, we also provide an alternative index page for beta tests, where anyone can enter the system by only inputting a preferred display name without registration. On logging in, each user also select team, either "“Team P1," or "Team P2."

Figure 1 shows the main page (after logging in), which can be vertically divided into 5 components. Component 1 on the top shows the name and team of the user, with a button for logging out. Component 2 contains embedded Twitch streaming content and chat area; in the streaming content, besides the game screen, texts are added for providing information such as character names and battle results. Component 3 shows two buttons: a green button for cheering (strengthen the AI of the user's team) and a red button for jeering (weaken the opponent AI). Component 4 shows a leaderboard, with the score of the user added on the bottom. Component 5 shows the number of users online; it tells how many members are there in each team.

This interface was implemented using a combination of web-programming languages. The client side - the log-in page 


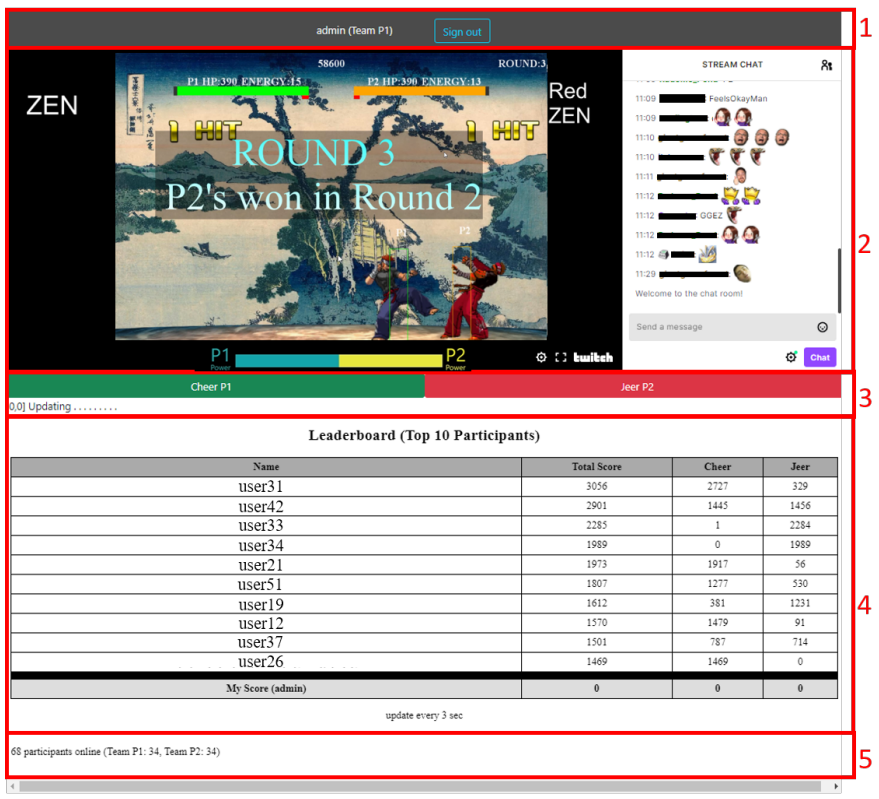

Fig. 2. A system screenshot of the system running on a web browser. The page can be vertically divided into 5 components: (1) display name and team of the current user, with a button for log out, (2) live-stream video and a chat area on the right, (3) buttons for cheer and jeer, (4) a leaderboard showing top-10 most active users, and (5) the number of users online.

and the container of all components in Fig. 1-was implemented by ReactJS. Contents for Component 4 and 5 updates every 3 and 10 seconds by the ReactJS page calling PHP (that contain HTML and JS codes) scripts on the server side-PHP scripts query data from an SQL database and render HTML components.

\section{B. Database with $O D S$}

Structured Query Language (SQL) is used for the game database. All interface usage data are kept. The database was designed to also serve as an operational data store $^{7}$ (ODS), e.g., there are tables containing information about the current user session and game round, containing counters to accelerate the process of leaderboard generation. There are a couple of reasons why SQL was chosen, some of which are as follows:

- No bandwidth limit: Twitch chat messages were used in some other systems (e.g., [11], [18], [22]), but our game systems has much larger data flow since each user can click many times in each second. We tried this method and the quota for message sending ran out in a few minutes with less than ten users.

- No additional cost: Firebase was tested. We found that, even if the size of data sent is small, the number of data sent is large. Such number is counted as "document writes" by Firebase ${ }^{8}$, and its quota ran out quickly in our testing, signalling a quick growth in the cost for Firebase upgrade.

7 a repository that stores a snapshot of current data for real-time processing ${ }^{8} \mathrm{https}: / /$ firebase.google.com/docs/firestore/quotas
TABLE II

VARIABLES USED IN THE GAME SYSTEM.

\begin{tabular}{|c|l|}
\hline variablename & definition \\
\hline $\begin{array}{c}\text { name } \\
\text { team }\end{array}$ & name of the user \\
$c 1$ & team of the user \\
$j 1$ & the number of cheers for P1 in the buffer \\
$c 2$ & the number of jeers for P1 in the buffer \\
$j 2$ & the number of cheers for P2 in the buffer \\
$o$ & the number of jeers for P2 in the buffer \\
$s$ & binary integer telling if the game is online \\
$r$ & id of the current session (group of users) \\
\hline
\end{tabular}

- Reduce unnecessary data temporarily stored in memory: SQL does not only overcome the above two problems, one additional benefit is that data from the interface go directly to the data storage, and the gameplay controller only needs to query and store a few amount of data needed for adjusting game contents.

- Distribute computation and unitize SQL query optimization: In our game system, each message takes effect for 15 seconds. Without SQL, it is needed to temporarily store click data in the controller (continuously adds and removes click data to a list for computation). With SQL, the controller left the computation to the SQL server and used a single optimized query to obtain only a summary of 15 -second data for processing.

- Allow quick access of data and allow real-time report on demand: generating reports from most recent data can be efficiently done, with flexibility from the use of SQL commands.

Table II shows variables stored in the interface and the gameplay controller. Algorithms 1 and 2 are used by the interface for updating Component 4 and 5 in Fig. 2 respectively. The database schema and SQL commands (SQLcmds) used to establish dataflow between system components, as well as a detailed version of algorithms, are available on osf.io9.

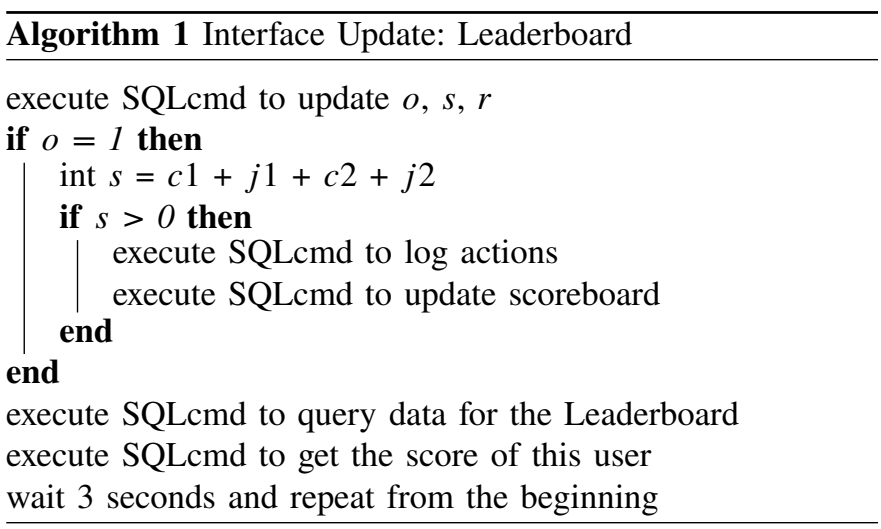

\section{Marionettist (Gameplay Controller)}

Marionettist is a component or sub-system responsible for controlling the strength of AI in the game, as well as updating the game server status (i.e., online and round_id).

\footnotetext{
${ }^{9}$ https://osf.io/gpb7w/wiki/1.\%20Database/
} 


\begin{tabular}{l}
\hline Algorithm 2 Interface Update: online status \\
\hline execute SQLcmd to remove offline users \\
execute SQLcmd to update the status of this user \\
execute SQLcmd to query online status of users \\
wait 10 seconds and repeat from the beginning \\
\hline
\end{tabular}

As aforementioned, the game system use two gBEAIs. The strength of each gBEAI is controlled by a parameter $F$. In Marionettist, a new parameter named "Dominance $(D)$ " is introduced-inspired by a study on a dominance adjustment $\mathrm{AI}$ by $\mathrm{Xu}$ et al. [31]. This parameter is simply equal to $-F_{1}$ and $-F_{1}$ (cf. Eq. 1), which means that its value is in the range of $[-1,1]$, positives values let $\mathrm{P} 1$ dominate the game (e.g., at the value of $1, \mathrm{P} 1$ is expected to win by $120 \mathrm{HP}$ difference), negative values let $\mathrm{P} 2$ dominate the game, otherwise equal match.

$$
D=-F^{P 1}=F^{P 2}
$$

Eq. 1 can be broken down to Eq. 2, where $k^{P 1}$ and $k^{P 2}$ are the total number of clicks favoring $\mathrm{P} 1$ and $\mathrm{P} 2$ respectively. $k^{P 1}$ can be either cheering P1 or jeering P2 (cf. Eq. 3), while $k^{P 2}$ can be either cheering P2 or jeering P1 (cf. Eq. 4). These inputs are obtained by executing SQLcmd to obtain data for updating $D$ every one second (cf. Algorithm 3).

$$
\begin{gathered}
D=\frac{k^{P 1}-k^{P 2}}{k}=\frac{k^{P 1}-k^{P 2}}{k^{P 1}+k^{P 2}} \\
k^{P 1}=\text { total_cheer_P1+total_jeer_P2} \\
k^{P 2}= \begin{cases}\text { total_cheer_P2+total_jeer_P1, } & \text { Competitive } \\
x \cdot \arg \min (t, \Delta), & \text { Collaborative }\end{cases}
\end{gathered}
$$

It is also noted that the game has two modes, where in Collaborative Game, there are no clicks supporting P2 from the users. Therefore, $k^{P 2}$ (cf. Eq. 4) is computed based on a parameter $x$ (cf. Eq. 5), which is an average number of clicks per user per second $\left(k_{r-1}\right.$ is the total number of clicks in the last round, while $n$ is the total number of users). This parameter $x$ is updated at the beginning of each game round using data obtained from SQLcmd. $k^{P 2}$ is computed by multiplying $x$ with the game time since the round begins in second $(t)$.

$$
x=\frac{k_{r-1} \cdot n}{60 \cdot n_{r-1}}
$$

\begin{tabular}{l}
$\overline{\text { Algorithm } 3 \text { Game Update: adjust AIs' strength }}$ \\
\hline execute SQLcmd to get data and calculate $D$ \\
wait 1 seconds and repeat from the beginning
\end{tabular}

\section{FightingICE and gBEAIs}

No modification was done on FightingICE, but the AI; gBEAI is needed for at least one side. The AI passively received a parameter telling the targeted HP difference. The gBEAI was slightly modified, only to make it send data about the game state to the Marionettist to notify when a game round begin and end.

Compared to the original FightingICE, there are texts added for showing characters' names as well as announcing the starting of a new round and a previous-round's result (cf. Figure 1), these texts are exported from the AI as text files. $\mathrm{OBS}^{10}$ reads these files and shows texts as parts of a streaming content. There is also a bar with blue and yellow color below the game screen telling which side of the player is dominating the game-this bar is provided by the Marrionettist.

\section{ExPeriment And Results: AI Test}

Similarly to our previous work [19], we first evaluated the performance of the proposed AI. Here, we investigate how long two gBEAIs would take to adjust their strengths and converge the targeted HP difference, when the value of $D$ changes.

\section{A. Apparatus}

The test was conducted on Fighting ver.4.5. "ZEN" were used on both sides; this is a default character whose parameters of skills are publicly accessible ${ }^{11}$. Settings of MCTS parameters were based on a study [10]. Dell Computers with Intel(R) Xeon(R) W-2135 CPU 3.70GHz 3.70GHz, RAM 16GB, Windows 10 Pro for Workstations x64 were used to run the tests.

\section{B. Settings and Results}

We considered 9 different settings of $D: D_{1}=0, D_{2}=$ $1, D_{3}=-1, D_{4}=0$ to $1{ }^{12}, D_{5}=0$ to $-1, D_{6}=1$ to 0 , $D_{7}=-1$ to $0, D_{8}=1$ to -1 , and $D_{9}=-1$ to 1 . It is noted that $D_{2} \& D_{3}, D_{4} \& D_{5}, D_{6} \& D_{7}$, and $D_{8} \& D_{9}$, are pairs with actually the same setting, but the sides of P1 and P2 are switched-each setting was tested this way because the side of characters possibly affect the AI's strength. Per setting, excepted $D_{1}$, the test consisted of 50000 rounds in Time Mode (the round time is fixed to 60 seconds, and HPs starts from 0 to negative values). The test on $D_{1}$ consists of 100000 rounds. After results from the same pair were combined, there were 5 settings of $D$ s left, each with data from 100000 rounds; these results are shown in Fig. 3. The HP difference is denoted as $\triangle H P$, computed as P1's HP subtracted by P2's HP.

A mean $\triangle H P$ reached around the targeted value in most cases at the middle and the end of the game. In the worst case, when $D=1$ to 1 , about 200 units of change in $\Delta H P$ could be achieved in the last 30 seconds, leaving 40 units different from the targeted value. More details and results about this AI test can be found on OSF ${ }^{13}$.

\footnotetext{
${ }^{10}$ an open source software used for Twitch live streaming

${ }^{11}$ ZEN Skill Table: https://www.ice.ci.ritsumei.ac.jp/ ftgaic/index-2a.html

${ }^{12}$ In each round, $D$ was set to 0 for the first 30 seconds, and switched to 1 for the last 30 seconds

${ }^{13}$ https://osf.io/6x2ep/
} 


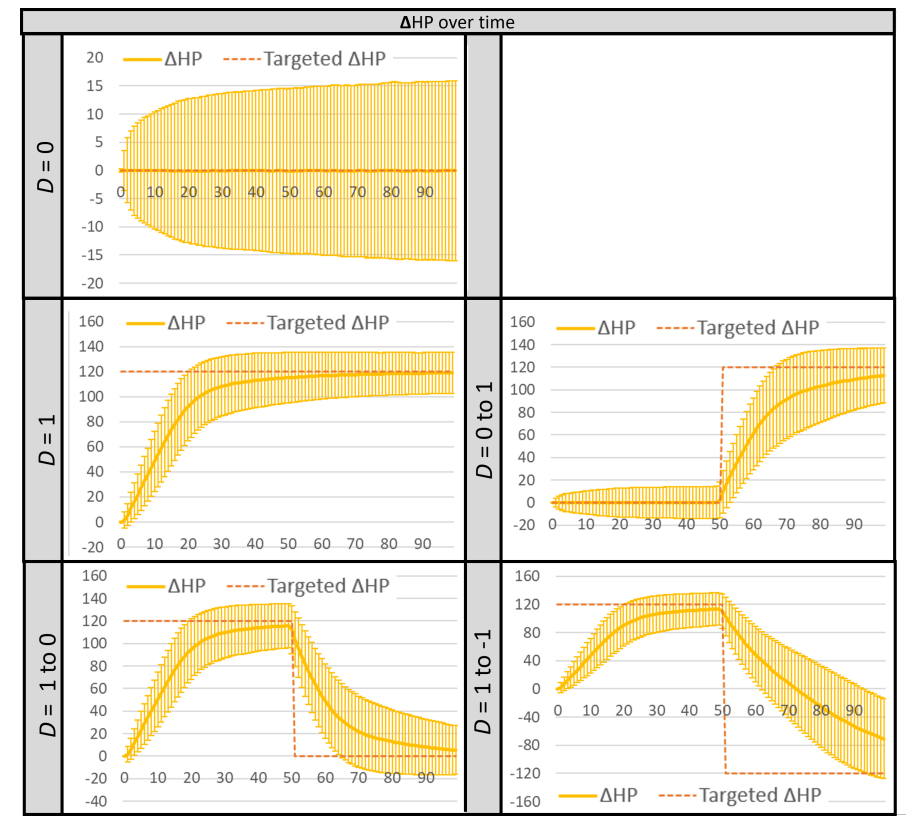

Fig. 3. Game test results for different settings of $D$; each was from a ten thousand 1-min rounds. $\triangle H P$ represents a mean with error bars of the absolute HP difference between the two characters. The x-axis represents the time point $(t)$, where $t=50$ and 100 are the middle and the end of a 1-min round.

\section{EXPERIMENT AND RESUltS: USER EXPERIMENT}

We conducted a user experiment to investigate how designs in our game affect user experience (UX) and activeness. Two different designs for audience participation- "Competitive" and "Collaborative"-were compared. "Watch-only," using Twitch without the interface, was added as a baseline (NonAPG). HP Mode ${ }^{14}$ was used, and ZEN was chosen as a character for both P1 and P2 (different character skins but same skills).

\section{A. Research Questions}

We are investigating areas that have not yet been wellstudied, and this study is exploratory research ${ }^{15}$. Six global research questions were formulated as follow:

1) Do APG and Non-APG affect UX differently?

2) Do Collaborative and Competitive games affect UX differently?

3) Do "Cheer" and "Jeer" affect UX differently?

4) Comparing collaborative and competitive games, do users cheer and jeer differently?

5) Are there any associations between activeness and UX?

6) Do users with different levels of familiarity with Twitch and fighting games perceive different UX?

Each of the above questions is to be answered in this study, not only "yes" or "no," but also "how," or "why." UX

${ }^{14}$ Each character has a $400 \mathrm{HP}$ at the beginning, and the round ends when HP of one character drops to 0 or timeout

${ }^{15} \mathrm{https}$ ://researcher-help.prolific.co/hc/en-gb/articles/360009500513-Am-Idoing-exploratory-or-confirmatory-research-Why-does-it-matter- will be analyzed in four independent gaming aspects: Play Engrossment, Enjoyment, Personal Gratification, and Social Connectivity. Experimental results will formulate hypotheses on what likely contributes to UX on each certain aspect; they are hypotheses expected to be tested and confirmed via experiments on games of various genres in the future. Following previous studies (e.g., [20]), we did not use Bonferroni correction due to the exploratory nature of the study.

\section{B. UX Assessment}

UX was assessed based on the Game User Experience Satisfaction Scale (GUESS) [21]. GUESS measures UX via several factors; a factor corresponds to a certain gaming aspect. Four applicable factors, namely Play Engrossment, Enjoyment, Personal Gratification, and Social Connectivity, were selected. Each of them was measured by asking three questions (those with the highest factor loadings from the original paper). Questions were slightly modified to fit our system.

In contrast to that the original GUESS uses "ranking (scaling)" as a self-reporting questionnaire scheme, our GUESS uses "pairwise preference." In our 4-AFC GUESS (cf. Table. III), users are asked to compare two given experimental conditions. This modification was based on findings from recent studies in game research (e.g., [32], [33]) that reporting via pairwise preferences has recently attracted the interest of researchers in affective and cognitive modeling as it minimizes the assumptions made about subjects' notions of highly subjective constructs such as emotions, reduces inter-personal differences, and eliminates artifacts such as the subjective notion of ranking/scaling. We modified GUESS questions using templates based on previous studies by Yannakakis et al. [33] and Burelli et al. [3].

\section{Candidates and Participants}

We recruited bachelor students from a class of "AI for Games" at Bangkok University; they had some prior knowledge about FightingICE since it was used as a case study in their class. Initially, there were a total of 161 candidates from the two class sessions (80 and 81). After removing those who were absent, disagreed to participate, did not complete either the consent form or the questionnaire, and those who did no action during the experiment, at the end, a total of 74 participants were left from the two sessions (39 and 35). The mean age of 62 participants who revealed their ages was 21.68 \pm 5.83 . There were 43 males, 3 females, and the rest are others or unrevealed. The two sessions become two groups for testing the two different designs: Competitive and Collaborative.

\section{Procedures}

The experiment was conducted remotely during the COVID19 pandemic via ZOOM cloud meeting. Procedures were as the followings.

1) Introduction and the informed consent: The experiment started with a 10-min introduction for providing details about the research study. During the first 5 minutes, a link for submitting an online informed consent was distributed, and 
TABLE III

4-AFC version of the Game User Experience Satisfaction Scale (GUESS)

\begin{tabular}{|l|l|}
\hline Factor & Questions \\
\hline $\begin{array}{l}\text { Play Engrossment } \\
(\mathbf{E g})\end{array}$ & $\begin{array}{l}\text { 1. In which game you felt more detached from the outside world while participating. } \\
\text { 2. In which game you cared less to check events that were happening in the real world during the game. } \\
\text { 3. In which game you were getting less tired while participating. }\end{array}$ \\
\hline $\begin{array}{l}\text { Enjoyment } \\
(\mathbf{E j})\end{array}$ & $\begin{array}{l}\text { 1. In which game you thought was more fun. } \\
\text { 2. In which game you more enjoyed participating } \\
\text { 3. In which game you felt more bored while participating. }\end{array}$ \\
\hline $\begin{array}{l}\text { Personal } \\
\text { Gratification (PG) }\end{array}$ & $\begin{array}{l}\text { 1. In which game you were more in suspense about whether your player would succeed. } \\
\text { 3. In which game you felt more successful when your player overcame the obstacles. }\end{array}$ \\
\hline $\begin{array}{l}\text { Social } \\
\text { Connectivity (SC) }\end{array}$ & $\begin{array}{l}\text { 1. In which game you wanted more to "cheer to help my player" / "jeer to hurt the opponent" as well as possible during the game. } \\
\text { 2. In which game you liked more to participate with otheraction between audiences. }\end{array}$ \\
\hline \begin{tabular}{l} 
3. In which game you were more able to participate with other audiences if you chose. \\
\hline
\end{tabular}
\end{tabular}

Four answer choices: (1) Game 1, (2) Game 2, (3) Both Equally, (4) Neither

items in it were explained. The last 5 minutes is given for them to quietly read the informed consent before submitting. Candidates were informed about details such as the purpose of this research study, how to play FightingICE, that their personal information will be removed, and that participation in this study is voluntary and they will not suffer disadvantageous countermeasures from disagreeing to participate. They were told that we were testing one of our designed APG interfaces, without details about interfaces used by other groups (i.e., we did not tell them that we are comparing Competitive and Collaborative). The informed consent was based on the research ethics guidelines at the authors' universities. According to the guidelines, our research does not require an ethics approval. The above form was provided in English with Thai translation (also the 4-AFC GUESS), and explanation is given in Thai. In total, there were 119 consents submitted, with 82 agreed to participate.

\section{E. Experiment and the questionnaire}

Both groups started with Watch-only for one game (three 1min rounds), took a break for 3 minutes (while details about the next game was given), and then participated in one APG (either Competitive or Collaborative), before proceeding to the questionnaire. In total, it was expected to take 19 minutes for the experiment, plus 5 minutes for the questionnaire.

\section{F. Results}

1) Frequencies of responses: Seventy five participants completed the questionnaire. Data of one participant was later removed as he did no action and gave the same answer to all the questions. Therefore, data from 74 participants were left for analysis. A frequency histogram showing raw responses from the GUESS questionnaire is shown in Figure 4, where Group I (G-I) were those participated in Watch-only and then Competitive Game, and Group II (G-II) were those participated in Watch-only and then Collaborative Game.

Pre-processing was done to combine answers from three questions to one "final answer," indicating each individual's preference for each certain UX factor. This step was simply done by taking the majority answers from the three questions, and in case there exists no preference, "unclear" is taken as the

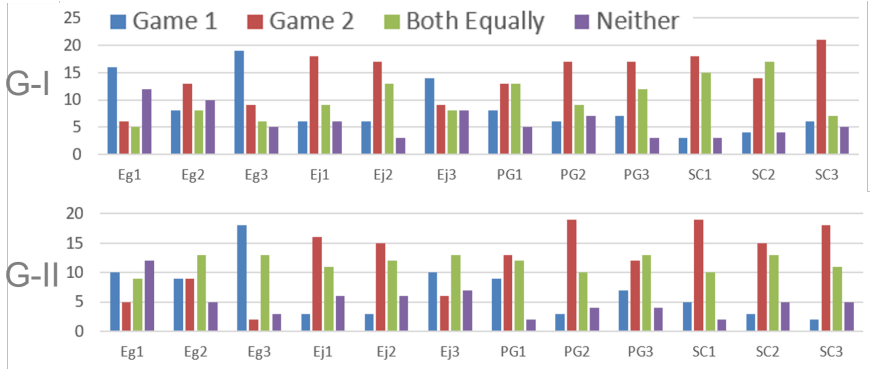

Fig. 4. Frequencies of responses from the GUESS questionnaire (raw results).

final answer (labeled as " 0 "). It is noted that the third question for assessing Enjoyment was a negative statement, while the others were positive, so answers 1 and 2, as well as 3 and 4, were switched for this question before taking the final answer. A frequency histogram of final answers is shown in Figure 5, and their values are given in Table IV.

2) Difference between experimental conditions: The first step of analysis was to run a chi-square goodness-of-fit test in SPSS Statistics on the above final answer data to check whether the obtained result is different from a result by chance. Namely, for each factor, there were a total of $4^{3}$ or 64 possible combinations of answers from the three question. If the result was obtained by chance, the proportions to obtain "Answer 0 " would be 24 , while the rest would be 10 each; these proportions led to in Table IV. The results told us that frequencies on all UX factors of both groups are significantly different from their expected values when all type of answers were considered (cf. Table V).

The next step focused on Answer 1 and 2 and checked whether two different game conditions, Non-APG and APG, affect UX differently. Binomial test (exact test of goodnessof-fit) was used; the two considered were compared with an expected ratio of 1:1. The results are shown in Table V; there existed differences between the two conditions on all the factors on both groups, except Engrossment on Group 1.

Taking into account frequencies in Figure 5, it can be concluded that APG (either Competitive or Collaborative) was more preferable than Non-APG on aspects of Enjoyment, 


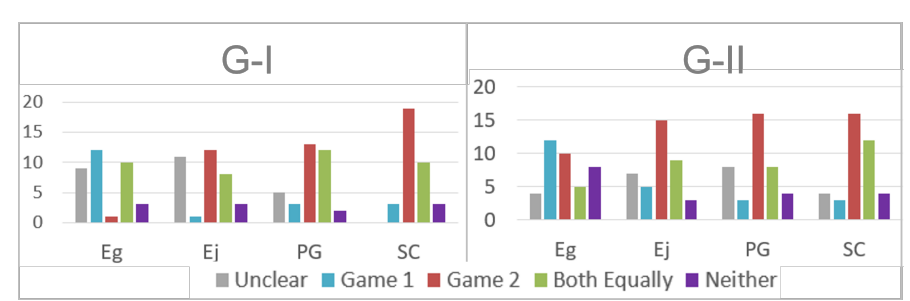

Fig. 5. Frequencies of final answers.

TABLE IV

PREFERENCES OF PARTICIPANTS BASED ON FOUR UX FACTORS.

\begin{tabular}{|l|c|c|cccc|}
\hline & Answer* & $\hat{\mathrm{n}}^{* *}$ & $\mathrm{Eg}$ & $\mathrm{Ej}$ & $\mathrm{PG}$ & $\mathrm{SC}$ \\
\hline Group 1 (G-I) & 0 & 14.6 & 4 & 7 & 8 & 4 \\
Competitive & 1 & 6.1 & 12 & 5 & 3 & 3 \\
$(N=39)$ & 2 & 6.1 & 10 & 15 & 16 & 16 \\
& 3 & 6.1 & 5 & 9 & 8 & 12 \\
& 4 & 6.1 & 8 & 3 & 4 & 4 \\
\hline Group 2 (G-II) & 0 & 13.1 & 9 & 11 & 5 & 0 \\
Collaborative & 1 & 5.5 & 12 & 1 & 3 & 3 \\
$(N=35)$ & 2 & 5.5 & 1 & 12 & 13 & 19 \\
& 3 & 5.5 & 10 & 8 & 12 & 10 \\
& 4 & 5.5 & 3 & 3 & 2 & 3 \\
\hline
\end{tabular}

*Answer: (0) Unclear, (1) Prefer Game 1, (2) Prefer Game 2, (3) Prefer Both Equally, (4) Prefer Neither

**n̂ : expected frequency assuming that the result was obtained by chance

Personal Gratification, and Social Connectivity. However, participants perceived significantly lower Engrossment on Collaborative APGs than Non-APG. On Competitive APGs, the similar perception was found, but it was much smaller and insignificant (12 for Non-APG vs for 10 Competitive APG).

We also ran a chi-square test for association to find correlations between group of participants and UX factors. A significant difference was found (cg. Table VI) on Engrossment with a $p$-value of .011 , which confirms the difference found above.

3) Association - Audience Types: Participants were classified into three types of audience based on their click logs. From a ratio of $\frac{\text { Cheer }}{\text { Cheer+Jeer }}$, each participant was classified as "Cheer Audience (Cheerer)," "Jeer Audience (Jeerer)," and "Neutral Audience (Neutral)," when the value is more than

TABLE V

P-VALUES FROM STATISTICAL TESTS (GOODNESS-OF-FIT)

\begin{tabular}{|l|c|cccc|}
\hline & Group & Eg & Ej & PG & SC \\
\hline Chi, goodness-of-fit, & G-I & $\mathbf{. 0 0 2}$ & $\mathbf{. 0 0 0}$ & $\mathbf{. 0 0 0}$ & $\mathbf{. 0 0 0}$ \\
comparing all choices & G-II & $*$ & $\mathbf{. 0 0 1}$ & $\mathbf{. 0 0 7}$ & $\mathbf{. 0 0 0}$ \\
\hline Binomial test, & G-I & .835 & $\mathbf{. 0 4 1}$ & $\mathbf{. 0 0 4}$ & $\mathbf{. 0 0 4}$ \\
comparing Game 1 and 2 & G-II & $\mathbf{. 0 0 3}$ & $\mathbf{. 0 0 3}$ & $\mathbf{. 0 2 1}$ & $\mathbf{. 0 0 1}$ \\
\hline
\end{tabular}

* $p$-value cannot be obtained since the frequency of "Answer 0 " is zero (the expected frequency is 13.1); the difference was explicit on observation.
0.666 , less than 0.333 , and in between, respectively.

Based on chi-square tests for association (cf. Table VI), we found no significant associations between type of audience and UX factors, and well as between the type and experimental group. Nevertheless, the numbers of Cheerer, Jeerer, and Neutral were 23, 11, 5 for Group 1, and 49, 16, and 9 for Group 2. From percentages, $28.20 \%$ of participants were Jeerer in Competitive Game, compared to $14.28 \%$ in Collaborative Game.

From this result, we conclude that Cheer and Jeer did not affect UX differently, but users might tend to Jeer more in Collaborative games where the opponent side does not involve humans.

4) Association - Activeness: Activeness indicates the degree of participation. To run association tests, activeness was measured and then discretized to create categorical variables. On each group, participants were ranked based on their user scores, or equivalently the total number of clicks-there was no duplicated rank in our data.

From rank data, we performed discretization and created two categorical variables. The first variable was "Top10," containing a binary telling whether a certain participant was one of the top ten, whose score was shown on the leaderboard at the end of experiment, or not. The second variable was "Rank Group," where all the participants in each experimental group were divided into five equal groups; 1 was the most active group, while five was the least active.

Based on chi-square tests for association (cf. Table VI), we found that top-10 and non-top-10 participants in Group 1 perceived Personal Gratification differently. From frequencies, we found that none of the top-10 participants prefer Game 1. In other words, it can be said that Competitive APG promoted Personal Gratification.

We also analyzed associations between Top10 (or Rank Group) and Type of Audience. However, no association was found. It implied that All types of audience existed at all levels of activeness, or all types of audience were equally active.

5) Association - Familiarity: There were two types of familiarity collected: those to Twitch and fighting games (denoted as FTG). Based on chi-square tests for association, we got two important findings as follows:

- On Group 1, familiarity to Twitch affected the perception of Social Connectivity. The left image in Figure 6 shows frequencies over this association; F1 - F4 indicate the level of familiarity from low to high. It can be seen that, when the level of familiarity increased, unclear preference and preference towards Non-APG decreased.

- On Group 2, familiarity to fighting games affected Engrossment. The right image in Figure 6 shows frequencies over this association. It can be seen that, as the level of familiarity increased, preference towards NonAPG decreased. Those who were familiar with fighting games tended to have unclear preferences or perceive no engrossment from both games. 


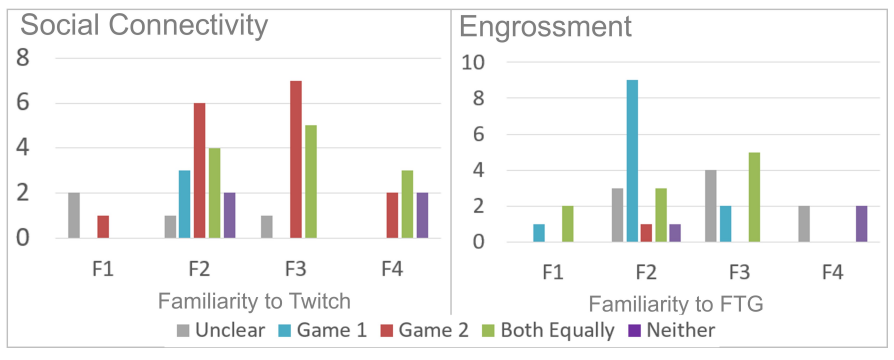

Fig. 6. Frequencies based on familiarity.

TABLE VI

P-VALUES From STATISTICAL TESTS (ASSOCIATIONS)

\begin{tabular}{|l|c|cccc|}
\hline & Group & Eg & Ej & PG & SC \\
\hline Group & - & $\mathbf{. 0 1 1}$ & .442 & .688 & .357 \\
\hline Type of Audience & Both & .283 & .899 & .468 & .254 \\
Type of Audience & G-I & .313 & .546 & .734 & .476 \\
Type of Audience & G-II & .757 & .116 & .713 & .084 \\
\hline Top10 & Both & .692 & .356 & .370 & .335 \\
Top10 & G-I & .652 & .711 &. $\mathbf{0 3 8}$ & .110 \\
Top10 & G-II & .636 & .545 & .228 & .989 \\
Rank Group & Both & .741 & .241 & .177 & .383 \\
Rank Group & G-I & .701 & .344 & .477 & .874 \\
Rank Group & G-II & .396 & .385 & .078 & .068 \\
\hline Familiarity to Twitch & Both & .675 & .060 & .663 & .352 \\
Familiarity to Twitch & G-I & .678 & .384 & .480 &. $\mathbf{0 4 7}$ \\
Familiarity to Twitch & G-II & .564 & .377 & .735 & .511 \\
Familiarity to FTG & Both & $\mathbf{. 0 4 6}$ & .089 & .862 & .829 \\
Familiarity to FTG & G-I & .639 & .501 & .837 & .733 \\
Familiarity to FTG & G-II & $\mathbf{. 0 4 5}$ & .433 & .505 & .622 \\
\hline
\end{tabular}

6) Additional results: Other findings from the questionnaires, game logs, and open-end questions, excluding positive comments and those irrelevant to APG, are as follows:

- During the competitive game, the two sides were almost equally strong. Team P1 barely won for 3 rounds. Nevertheless, win-lose did not significantly affect UX.

- Eighteen participants, or one-fourth, mentioned about "cheating"; they said that users who have a mouse that can assign macros ${ }^{16}$, or has an auto clicker, could cheat. Some participants suggested adding a cooldown between clicks.

\section{G. Limitations}

We learned that handling cheating and reducing inequality from devices are important points we missed. We investigated and found very high numbers of clicks on some 3-second intervals (more than 100). However, we did not remove participants who make these numbers because of four reasons: (1) the

${ }^{16}$ sequences of events (such as mouse clicks) that can be recorded and later played back to help perform repetitive tasks average numbers of click distributes at in all ranges, so it is difficult to determine a cut point, (2) those with high numbers of clicks did not have a consistent pattern, namely their clicks were not always high, the numbers decreased on some period, so we could not confidently label cheaters, (3) those with not too high number numbers could cheat as well, and (4) those with high clicks might not actually cheat, but used a touchscreen device that allowed them to click using multiple fingers. Nevertheless, besides the need for a mechanism to prevent cheating or reducing inequality from devices, this told us that a reliable measurement of Activeness can be difficult, and hence the types of user may not be reliably classified. In addition, we found that auto clickers and macros became widely known as there were recently a viral click game a few months before the experiment ${ }^{17}$.

Another limitation is varieties in audience demographics and consideration of this factor on analyses. We use participants with similar profiles to focus on effects from kinds of interaction and gameplays. We did not consider gender and age, although we have these data. For gender, we did not see the need to since our game content targets mass public, not personalized content. In addition, we did not have enough data from LGBTQ+ to analyze their preferences. Age was not considered because most participants have a similar age, with some outliers. Future studies may consider participants with more varieties of demographics.

\section{CONCLUSION AND Future Works}

This paper presented an audience participation fighting game, based on FightingICE and Twitch. Design and architecture of the system are discussed. Two types of gameplay, collaborative and competitive games, as well as two types of interaction, cheering and jeering, were presented and compared. The proposed system was evaluated on AI tests, and user experiment was then conducted to investigate how types of gameplay and types of interaction affect UX and their activeness.

The results provide clues on what may affect UX in the APG environment. Since we only tested with one game genre, instead of drawing conclusions, we here formulated hypotheses for future research to test on various game genres. From research questions in Section 4, we did an experiment and got results leading to the following hypotheses. [H1] allowing audiences to participate, regardless of whether in a competitive or collaborative environment, can enhance their Enjoyment, Personal Gratification, and Social Connectivity. However, Engrossment may decrease in a collaborative environment. [H2] competitive and collaborative games affect Engrossment differently. [H3] Cheering and Jeering do not differently affect UX in any aspect. [H4] audiences tend to jeer more in a competitive game, yet not statistically significantly. [H5] In a competitive environment, top players (most active players) whose names are on a leaderboard likely perceive a high

${ }^{17}$ https://asia.nikkei.com/Business/Media-Entertainment/Thailandjockeying-for-world-supremacy-in-a-cat-game 
Personal Gratification (but activeness might not be reliably assessed merely using a number of clicks). [H6] Familiarity with Twitch and the genre of the game of interest affects UX in some aspects.

Future work should focus on preventing cheating. Another challenge is to improve the AI strength adjustment. Related research may apply our proposed system architecture, as well as designs for interaction, to games of other genres. Studying UX of human players, on Human vs AI, as previously done in non-APG games (e.g., [1], [12], [14]), is also one promising direction. Finally, to allow reproducibility, supplementary information and files are provided on OSF 18

\section{REFERENCES}

[1] Nicholas David Bowman, Rene Weber, Ron Tamborini, and John Sherry. Facilitating game play: How others affect performance at and enjoyment of video games. Media Psychology, 16(1):39-64, 2013.

[2] Garland P Brooks and Ronald W Johnson. Floyd allport and the master problem of social psychology. Psychological Reports, 42(1):295-308, 1978.

[3] Paolo Burelli, Georgios Triantafyllidis, and Ioannis Patras. Non-invasive player experience estimation from body motion and game context. In 2014 IEEE Conference on Computational Intelligence and Games, pages 1-7. IEEE, 2014.

[4] Teresa Cerratto-Pargman, Chiara Rossitto, and Louise Barkhuus. Understanding audience participation in an interactive theater performance. In Proceedings of the 8th Nordic Conference on Human-Computer Interaction: Fun, Fast, Foundational, pages 608-617, 2014.

[5] Jie Deng, Gareth Tyson, Felix Cuadrado, and Steve Uhlig. Internet scale user-generated live video streaming: The twitch case. In International Conference on Passive and Active Network Measurement, pages 60-71. Springer, 2017.

[6] John Downs, Frank Vetere, Steve Howard, Steve Loughnan, and Wally Smith. Audience experience in social videogaming: effects of turn expectation and game physicality. In Proceedings of the SIGCHI Conference on Human Factors in Computing Systems, pages 3473-3482, 2014.

[7] John Downs, Frank Vetere, and Wally Smith. Differentiated participation in social videogaming. In Proceedings of the Annual Meeting of the Australian Special Interest Group for Computer Human Interaction, pages 92-100, 2015.

[8] Katharina Emmerich and Maic Masuch. Watch me play: does social facilitation apply to digital games? In Proceedings of the $2018 \mathrm{CHI}$ Conference on Human Factors in Computing Systems, pages 1-12, 2018.

[9] Oliver L Haimson and John C Tang. What makes live events engaging on facebook live, periscope, and snapchat. In Proceedings of the 2017 CHI conference on human factors in computing systems, pages 48-60, 2017.

[10] Makoto Ishihara, Suguru Ito, Ryota Ishii, Tomohiro Harada, and Ruck Thawonmas. Monte-carlo tree search for implementation of dynamic difficulty adjustment fighting game ais having believable behaviors. In 2018 IEEE Conference on Computational Intelligence and Games (CIG), pages 1-8. IEEE, 2018.

[11] Thanat Jumneanbun, Sunee Sae-Lao, Pujana Paliyawan, Ruck Thawonmas, Kingkarn Sookhanaphibarn, and Worawat Choensawat. Rap-style comment generation to entertain game live streaming. In 2020 IEEE Conference on Games (CoG), pages 706-707. IEEE, 2020.

[12] Dennis L Kappen, Pejman Mirza-Babaei, Jens Johannsmeier, Daniel Buckstein, James Robb, and Lennart E Nacke. Engaged by boos and cheers: the effect of co-located game audiences on social player experience. In Proceedings of the first ACM SIGCHI annual symposium on Computer-human interaction in play, pages 151-160, 2014.

[13] Mehdi Kaytoue, Arlei Silva, Loïc Cerf, Wagner Meira Jr, and Chedy Raiissi. Watch me playing, i am a professional: a first study on video game live streaming. In Proceedings of the 21st international conference on world wide web, pages 1181-1188, 2012.
[14] Charles E Kimble and Jeffery S Rezabek. Playing games before an audience: Social facilitation or choking. Social Behavior and Personality: an international journal, 20(2):115-120, 1992.

[15] Pascal Lessel, Alexander Vielhauer, and Antonio Krüger. Expanding video game live-streams with enhanced communication channels: a case study. In Proceedings of the 2017 CHI conference on human factors in computing systems, pages 1571-1576, 2017.

[16] Holin Lin and Chuen-Tsai Sun. The role of onlookers in arcade gaming: Frame analysis of public behaviours. Convergence, 17(2):125-137, 2011.

[17] Bernhard Maurer, Ilhan Aslan, Martin Wuchse, Katja Neureiter, and Manfred Tscheligi. Gaze-based onlooker integration: Exploring the inbetween of active player and passive spectator in co-located gaming. In Proceedings of the 2015 Annual Symposium on Computer-Human Interaction in Play, pages 163-173, 2015.

[18] Ngoc Cuong Nguyen, Ruck Thawonmas, Pujana Paliyawan, and Hai V Pham. Justin: An audience participation game with a purpose for collecting descriptions for artwork images. In 2020 IEEE Conference on Games (CoG), pages 344-350. IEEE, 2020.

[19] Pujana Paliyawan, Kingkarn Sookhanaphibarn, Worawat Choensawat, and Ruck Thawonmas. Towards social facilitation in audience participation games: Fighting game ais whose strength depends on audience responses. In 2020 IEEE Conference on Games (CoG), pages 686-689. IEEE, 2020.

[20] PM Pascoal, CF Raposo, and LB Oliveira. Predictors of body appearance cognitive distraction during sexual activity in a sample of men with ed. International journal of impotence research, 27(3):103-107, 2015.

[21] Mikki H Phan, Joseph R Keebler, and Barbara S Chaparro. The development and validation of the game user experience satisfaction scale (guess). Human Factors, 58(8):1217-1247, 2016.

[22] Dennis Ramirez, Jenny Saucerman, and Jeremy Dietmeier. Twitch plays pokemon: a case study in big g games. In Proceedings of DiGRA, pages 3-6, 2014.

[23] Joseph Seering, Saiph Savage, Michael Eagle, Joshua Churchin, Rachel Moeller, Jeffrey P Bigham, and Jessica Hammer. Audience participation games: Blurring the line between player and spectator. In Proceedings of the 2017 Conference on Designing Interactive Systems, pages 429-440, 2017.

[24] Max Sjöblom, Maria Törhönen, Juho Hamari, and Joseph Macey. The ingredients of twitch streaming: Affordances of game streams. Computers in Human Behavior, 92:20-28, 2019.

[25] Alina Striner, Andrew M. Webb, Jessica Hammer, and Amy Cook. Mapping design spaces for audience participation in game live streaming. In CHI '21: Proceedings of the 2021 CHI Conference on Human Factors in Computing Systems, pages 1-15, 2021.

[26] Michael J Strube. What did triplett really find? a contemporary analysis of the first experiment in social psychology. The American journal of psychology, pages 271-286, 2005.

[27] Norman Triplett. The dynamogenic factors in pacemaking and competition. The American journal of psychology, 9(4):507-533, 1898.

[28] Rina R Wehbe and Lennart E Nacke. Towards understanding the importance of co-located gameplay. In Proceedings of the 2015 Annual Symposium on Computer-Human Interaction in Play, pages 733-738, 2015.

[29] Donghee Yvette Wohn and Guo Freeman. Live streaming, playing, and money spending behaviors in esports. Games and Culture, 15(1):73-88, 2020.

[30] Tim Wulf, Frank M Schneider, and Stefan Beckert. Watching players: An exploration of media enjoyment on twitch. Games and culture, 15(3):328-346, 2020

[31] Junjie Xu. Player dominance adjustment in games. arXiv preprint arXiv:2110.11225, 2021.

[32] Georgios N Yannakakis and John Hallam. Ranking vs. preference: a comparative study of self-reporting. In International conference on affective computing and intelligent interaction, pages 437-446. Springer, 2011.

[33] Georgios N Yannakakis and Héctor P Martínez. Ratings are overrated! Frontiers in ICT, 2:13, 2015.

[34] Shubu Yoshida, Makoto Ishihara, Taichi Miyazaki, Yuto Nakagawa, Tomohiro Harada, and Ruck Thawonmas. Application of monte-carlo tree search in a fighting game ai. In 2016 IEEE 5th Global Conference on Consumer Electronics, pages 1-2. IEEE, 2016.

[35] Robert B Zajonc. Social facilitation. Science, 149(3681):269-274, 1965.

${ }^{18} \mathrm{https} / / / \mathrm{osf} . i o / v e f r d /$ 